\title{
Analysis of Electromagnetic Characteristics of Magnetohydrodynamic Rotator for Liquid Metals
}

\author{
Viktor N. Timofeev ${ }^{1,2}$, Natalia S. Shakhoval ${ }^{1,2}$, Maksim Y. Khatsayuk ${ }^{1,2}$, \\ Svetlana V. Kurnaeva ${ }^{1}$, Eduard R. Vinter ${ }^{1,2}$ \\ ${ }^{1}$ Siberian Federal University, Krasnoyarsk, Russia \\ 2 "RPC Magnetic Hydrodynamics" Ltd, Krasnoyarsk, Russia
}

\begin{abstract}
There has been set and analytically solved task on calculation of the electromagnetic field in the area of the "cylindric inductor - channel with liquid metal" system. The inductor part includes a polyphase winding with electric current that enables generating the rotating magnetic field. The solution for electromagnetic field vector has been found in the Fourier series of a complex form using Bessel functions. The task was solved in a $2 \mathrm{D}$ design using the cylindrical coordinate system. There have been obtained the relations of the current density in the channel metal, electromagnetic power and moment to the parameters and modes of system operation. The electromagnetic rotator for the liquid metal can be used to introduce fine metal particles (chips, sawdust) into the reverberatory melting furnace.
\end{abstract}

Index Terms - Electromagnetic rotator, electromagnetic field, differential equations, cylindrical coordinate system, Fourier series in a complex form, Bessel function.

\section{INTRODUCTION}

E VERY YEAR there has been observed a high increase in the use of aluminium alloys in a variety of industries. Improving the mechanical properties of an alloy produced can be achieved by adding various alloying agents. Different process flowsheets and equipment are used for their production [1]. In most cases aluminium alloys are produced in holding furnaces. The magnetohydrodynamic (MHD) rotator can be used for mixing small particles into the melt.

Fig. 1 shows the section of holding furnace with MHD rotator installed. The rotator consists of a magnetic core 4 and several coils of inductor winding 3. Mixing fine particles into melt 2 is performed in MHD rotator due to rotational movement of metal. In the center of the rotator the movement is directed downwards, this leads to alloying agents penetrating into the melt and then the melt with already mixed particles is fed to the lower layers of the melting furnace via connecting channel 5.

MHD rotator can be used in the processing of fine aluminium chips that are formed in the result of mechanical treatment of ingots. High quality metal output can be achieved when the chips are in the minimum time of contact with the hot surface of the melt. This is due to the fact that surface oxidation occurs in hot oxygenated air. The longer chips are on the melt surface, the thicker is the oxide layer and this, in its turn, complicates the separation of oxides from the metal. The metal cannot be smelt from chips and its output is significantly reduced. A prospecting direction of improving the efficiency of equipment for melting fine recycled aluminum (sawdust, chips, foils, etc.) is to create a funnel in the doghouse of a melting furnace. The funnel will provide for the immersion of recycled aluminum inside the melt in the melting furnace [2].

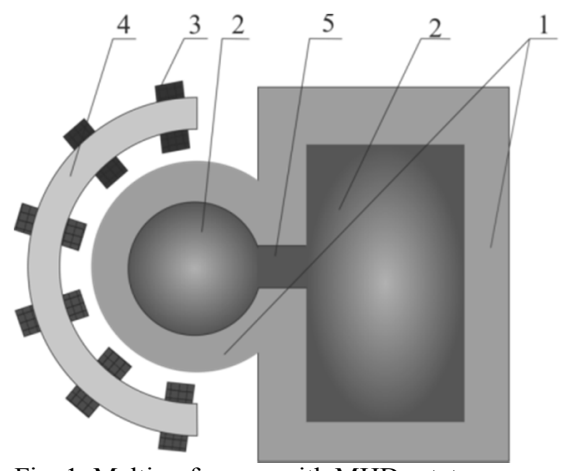

Fig. 1. Melting furnace with MHD rotator

1 is lining of furnaces and MHD rotator;

2 is melt (liquid metal);

3 is coil of inductor winding of MHD rotator;

4 is magnetic core (core) of MHD rotator ;

5 is channel connecting the MHD rotator with the bath of the melting furnace.

Currently, many studies on the creation of liquid metal motion by means of a magnetic field have been conducted, numerical and experimental studies have been conducted [3-8]. In this paper we will consider the electromagnetic characteristics of the rotator, creating a rotational and translational motion of the melt.

\section{PROBLEM STATEMENT}

To build the design model of the "cylindrical inductor channel" system there have been adopted the following assumptions.

1. We adopt the surfaces of melt and magnetic core of MHD rotator as cylindrical with radius $r$ (melt) and $\mathrm{R}$ (magnetic core).

2. The winding of the inductor is presented in the form of surface currents with a complex linear density $\dot{j}_{m}$ 


$$
\dot{j}_{m}=j_{m} e^{i \alpha_{m}}
$$

where $\alpha_{m}$ is a current phase in $m$-th coil;

$$
j_{m}=\frac{W_{m} I_{m}}{2 \Delta \varphi R}
$$

is the value of linear current load on the m-th coil.

Here $2 \Delta \varphi$ is the arc angle of -th layer in the cylindrical coordinate system $(\rho, \varphi) ; W_{m} I_{m}$ is a number of turns and current of $\mathrm{m}$-th coil.

3. We consider the coordinate system as strictly related to the inductor and the melt rotates with the constant angular velocity $\Omega$ as a solid body.

4. The magnetic core of the inductor is replaced with the media of magnetic permeability $\mu=\infty$ and electric conductivity $\gamma=0$, which restricts the design area with the surface of magnetic core $\rho=R$.

5. The design model has infinite size along the $Z$ axis, i.e. electro-magnetic field is a plane-parallel.

In the cylindrical coordinate system $(\rho, \varphi, z)$, the complex strengths of electric and magnetic field comprises as follows:

$$
\dot{\bar{E}}=\bar{e}_{z} \dot{E}_{z}, \quad \dot{\bar{H}}=\bar{e}_{\rho} \dot{H}_{\rho}+\bar{e}_{\varphi} \dot{H}_{\varphi} .
$$

The design model has two areas (Fig. 2):

Area $1(\rho<r)$ is characterized with the specific electric conductivity $\gamma$ and magnetic permeability $\mu_{0}$ and rotates clockwise with a constant angular velocity $\Omega$.

Area $2(r \leq \rho \leq R)$ has a specific electric conductivity $\gamma=0$, magnetic permeability $\mu_{0}$ and is limited to the ferromagnet cylindric medium $\rho>\mathrm{R}$ with $\gamma=0$ and $\mu_{0}=\infty$.

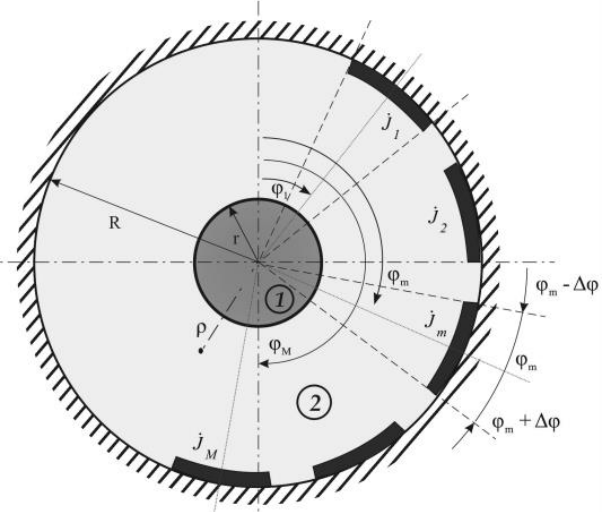

Fig. 2. Design model.

The ferromagnetic surface $(\rho=R)$ bears $M$ infinitely thin layers of current layers of the complex linear density $\mathrm{J}_{\mathrm{m}}$ (see Fig.2). The length of each $\mathrm{m}$-th layer is equal to $2 \mathrm{R} \Delta \varphi$.

The boundary problem is in finding the tensions of electric and magnetic fields in the cylindrical coordinate system that satisfy Maxwell equations and the relevant boundary conditions in areas 1 and 2 .

\section{THEORY}

\section{A. Electromagnetic field equations and boundary condition}

Directly from Maxwell's equations we can obtain differential equations $\dot{E}_{z}$ in areas 1 and 2. [8]

In area $1(\rho<r)$ :

$$
\begin{aligned}
& \frac{\partial^{2} \dot{E}_{z 1}}{\partial \rho^{2}}+\frac{1}{\rho} \frac{\partial \dot{E}_{z 1}}{\partial \rho}+\frac{1}{\rho^{2}} \frac{\partial^{2} \dot{E}_{z 1}}{\partial \varphi^{2}}, \\
& +\mu_{0} \gamma \cdot \Omega \frac{\partial \dot{E}_{z 1}}{\partial \varphi}-i \omega \mu_{0} \gamma \dot{E}_{z 1}=0
\end{aligned}
$$

where $\omega=2 \pi f$ is the angular current frequency in circuits,

$$
\begin{aligned}
& \mu_{0}=2 \pi \cdot 10^{-7} \mathrm{H} / \mathrm{m}, \\
& i=\sqrt{-1} . \\
& \text { In area } 2(\mathrm{r} \leq \rho \leq \mathrm{R}): \\
& \quad \frac{\partial^{2} \dot{E}_{z 2}}{\partial \rho^{2}}+\frac{1}{\rho} \frac{\partial \dot{E}_{z 2}}{\partial \rho}+\frac{1}{\rho^{2}} \frac{\partial^{2} \dot{E}_{z 2}}{\partial \varphi^{2}}=0 .
\end{aligned}
$$

At the boundary of areas 1 and $2(\rho=r)$ the following boundary conditions are true:

$$
\begin{gathered}
\dot{E}_{z 1}(r, \varphi)=\dot{E}_{z 2}(r, \varphi), \\
\dot{H}_{\varphi 1}(r, \varphi)=\dot{H}_{\varphi 2}(r, \varphi) .
\end{gathered}
$$

Maxwell equation $\operatorname{rot} \bar{E}=-i \omega \mu_{0} \bar{H}$ imply that

$$
\begin{gathered}
\dot{H}_{\varphi}=\frac{1}{i \omega \mu_{0}} \frac{\partial \dot{E}_{z}}{\partial \rho}, \\
\dot{H}_{\rho}=-\frac{1}{i \omega \mu_{0}} \frac{\partial \dot{E}_{z}}{\partial \varphi} .
\end{gathered}
$$

The following edge condition is observed on the surface of the magnetic circuit $(\rho=R)$ :

$$
\dot{H}_{\varphi 2}=\left\{\begin{array}{l}
-j_{m}, \varphi_{m}-\Delta \varphi_{m}<\varphi<\varphi_{m}+\Delta \varphi_{m} \\
0 .
\end{array}\right.
$$

Substituting expression (5) into (4) and (7), we obtain the following

$$
\begin{gathered}
\frac{\partial \dot{E}_{1}}{\partial \rho}(r)=\frac{\partial \dot{E}_{2}}{\partial \rho}(r), \\
\frac{\partial \dot{E}_{2}}{\partial \rho}(R)= \\
=\left\{\begin{array}{l}
-i \omega \mu_{0} j_{m}, \varphi_{m}-\Delta \varphi_{m}<\varphi<\varphi_{m}+\Delta \varphi_{m}, \\
0 .
\end{array}\right.
\end{gathered}
$$

\section{B. Solving electromagnetic field equations}

We search for the solution of differential equations (1) and (2) in the form of Fourier series in the complex form ( $\mathrm{z}$ index is further omitted). [10]

$$
\dot{E}_{1,2}(\rho, \varphi)=\sum_{n=-\infty}^{n=+\infty} \dot{E}_{n 1,2}(\rho) \cdot e^{i n \varphi},
$$


where

$$
\dot{E}_{n 1,2}(\rho)=\frac{1}{2 \pi} \int_{0}^{2 \pi} \dot{E}_{1,2}(\rho, \varphi) \cdot e^{-i n \varphi} d \varphi .
$$

Zero terms in Fourier series $(\mathrm{n}=0)$ must be excluded since $\sum_{m=1}^{M} \dot{J}_{m}=0$.

Multiplying equations (1) and (2) by the function $\frac{1}{2 \pi} e^{-i n \varphi}$ and integrating the resulting expression by $\varphi$ from 0 to $2 \pi$, we obtain

$$
\begin{gathered}
\frac{1}{\rho} \frac{\partial}{\partial \rho}\left(\rho \frac{\partial \dot{E}_{n 1}}{\partial \rho}\right)+\left(\beta_{n}^{2}-\frac{n^{2}}{\rho^{2}}\right) \dot{E}_{n 1}=0, \\
\frac{1}{\rho} \frac{\partial}{\partial \rho}\left(\rho \frac{\partial \dot{E}_{n 2}}{\partial \rho}\right)-\frac{n^{2}}{\rho^{2}} \dot{E}_{n 2}=0,
\end{gathered}
$$

where $\beta_{n}^{2}=i\left(n \mu_{0} \gamma . \Omega-\mu_{0} \gamma \omega\right)$.

Common solutions of ordinary differential equations of the 2 nd order (12) and (13) have the form [10]:

$$
\begin{gathered}
\dot{E}_{n 1}=C_{n 1} F_{n}\left(\beta_{n} \rho\right)+D_{n 1} \Phi_{n}\left(\beta_{n} \rho\right), \\
\dot{E}_{n 2}=C_{n 2} \rho^{n}+D_{n 2} \rho^{n},
\end{gathered}
$$

Where $F_{n}\left(\beta_{n} \rho\right)=J_{n}\left(\beta_{n} \rho\right)$ and $\Phi_{n}\left(\beta_{n} \rho\right)=I_{n}\left(\beta_{n} \rho\right)$ are an ordinary and modified Bessel functions of the n-th order from the argument $\beta_{\mathrm{n}}$ and $\rho$. [9]

Here the constant $D_{n 1}$ has to be equated to zero since subject to $\rho$ tending to zero, the function $\Phi_{n}\left(\beta_{n} \rho\right)$ tends to infinity. Boundary conditions (3) and (8) are valid for $\dot{\mathrm{E}}_{n 1}$ and $\dot{\mathrm{E}}_{n 2}$.

Multiplying equations (3), (8) and (9) by the function $\frac{1}{2 \pi} e^{-i n \varphi}$ and integrating the resulting expression by $\varphi$ from 10 to $2 \pi$, we obtain

$$
\begin{gathered}
\dot{E}_{n 1}(r)=\dot{E}_{n 2}(r) ; \\
\frac{\partial \dot{E}_{n 1}}{\partial \rho}(r)=\frac{\partial \dot{E}_{n 2}}{\partial \rho}(r) .
\end{gathered}
$$

We also convert boundary condition (9) in compliance with (10) and (11). Multiplying (9) by the function $\frac{1}{2 \pi} e^{-i n \varphi}$ and integrating the resulting expression by $\varphi$ from 0 to $2 \pi$, we will have:

$$
\frac{\partial \dot{E}_{n 2}}{\partial \rho}(R)=\eta_{n}
$$

Here

$$
\begin{gathered}
\eta_{n}=-\frac{i \omega \mu_{0}}{2 \pi} \sum_{m=1}^{M} \int_{\varphi_{m}-\Delta \varphi_{m}}^{\varphi_{m}+\Delta \varphi_{m}} \dot{\mathrm{j}}_{m} e^{-i n \varphi} d \varphi= \\
=-i \frac{\omega \mu j}{n \pi} N_{n 1},
\end{gathered}
$$

where

$$
\begin{gathered}
N_{n 1}=\sum_{m=1}^{M} \hat{j}_{m} e^{-i n \varphi_{m}} \sin \Delta \varphi_{m}, \\
\hat{j}_{m}=\frac{j_{m}}{j_{b}} .
\end{gathered}
$$

Placing (14) and (15) in the boundary conditions (16), (17) and (18), we obtain the system of equations

$$
\left\{\begin{array}{ccc}
0 & +C_{n 2} R^{n-1}-D_{n 2} R^{n-1} & =\frac{\eta_{n}}{n} \\
C_{n 1} F_{n}\left(\beta_{n} r\right) & -C_{n 2} r^{n}-D_{n 2} r^{-n} & =0 \\
C_{n 1} \frac{\beta_{n}}{n} F_{n}^{\prime}\left(\beta_{n} r\right) & -C_{n 2} r^{n-1}+D_{n 2} r^{-n-1} & =0 .
\end{array}\right.
$$

Solving the system of algebraic equations (19) on the constant $C_{n 1}, C_{n 2}$ and $D_{n 2}$ and placing them in (14) and (15), we obtain

$$
\begin{gathered}
\dot{E}_{n 1}=\frac{2 R \eta_{n}}{Q_{n}} ; \\
\dot{E}_{n 2}=R \eta_{n} \frac{G_{n}(\rho)}{n Q_{n}},
\end{gathered}
$$

where

$$
\begin{aligned}
& G_{n}(\rho)=n F_{n}\left(\beta_{n} r\right)\left[\left(\frac{\rho}{r}\right)^{n}+\left(\frac{\rho}{r}\right)^{-n}\right]+ \\
& +r \beta_{n} F_{n}^{\prime}\left(\beta_{n} r\right)\left[\left(\frac{\rho}{r}\right)^{n}-\left(\frac{\rho}{r}\right)^{-n}\right] \\
& Q_{n}=n F_{n}\left(\beta_{n} r\right)\left[\left(\frac{R}{r}\right)^{n}-\left(\frac{R}{r}\right)^{-n}\right]+ \\
& +r \beta_{n} F_{n}^{\prime}\left(\beta_{n} r\right)\left[\left(\frac{R}{r}\right)^{n}+\left(\frac{R}{r}\right)^{-n}\right] .
\end{aligned}
$$

Placing the obtained expressions for $E_{n 1}$ and $E_{n 2}$ into the Fourier series (11), we record the required solutions for the electric field efforts

$$
\begin{aligned}
& \dot{E}_{1}(\rho, \varphi)=-2 i \omega \mu_{0} R \frac{j}{\pi} \sum_{n=-\infty}^{n=+\infty} \frac{N_{n 1}}{n} \cdot \frac{F_{n}\left(\beta_{n} \rho\right)}{Q_{n}} e^{i n \varphi} \\
& \dot{E}_{2}(\rho, \varphi)=-i \omega \mu_{0} R \frac{j}{\pi} \sum_{n=-\infty}^{n=+\infty} \frac{N_{n 1}}{n^{2}} \cdot \frac{G_{n}(\rho)}{Q_{n}} e^{i n \varphi} .
\end{aligned}
$$

\section{Expressions for the current density in the channel and electromagnetic moment}

In a coordinate system fixed relative to the coil, the electrical field effort in the rotating channel with the angular velocity $\Omega$ is equal to

$$
\dot{E}_{z 1}^{\prime}=\dot{E}_{1 z}+\mu_{0} \Omega \rho \dot{H}_{\rho 1} .
$$

Here $\dot{\mathrm{E}}_{1 z}$ is generated by the electromotive force induced by the alternating magnetic field, penetrating liquid metal EMF of transformation. The addend is the effort of the 
electrical field caused by the metal rotation in a magnetic field - EMF of movement.

We define expression $\dot{H}_{\rho 1}$ from (6) subject to (20), we obtain

$$
\begin{gathered}
\dot{H}_{\rho 1}=-\frac{1}{i \omega \mu_{0} \rho} \frac{\partial \dot{E}_{z 1}}{\partial \varphi}= \\
=i \frac{2 R}{\rho} \sum_{n=-\infty}^{n=+\infty} \frac{N_{n 1} G_{n}(\rho)}{n Q_{n}} e^{i n \varphi}
\end{gathered}
$$

Placing the resulting expression (32) into (31), we will have

$$
\begin{gathered}
\dot{E}_{z 1}^{\prime}=- \\
2 i \omega \mu_{0} R \sum_{n=-\infty}^{n=+\infty} \frac{N_{n 1} G_{n}(\rho)}{n Q_{n}} \times \\
\times\left(1-n \frac{\Omega}{\omega}\right) \cdot e^{i n \varphi} .
\end{gathered}
$$

The current density in the channel is determined by Ohm's law in the differential form [10]

$$
\begin{gathered}
\delta(\rho, \varphi)=\gamma \dot{E}_{z 1}^{\prime}=. \\
=-2 i \omega \mu_{0} R \gamma \sum_{n=-\infty}^{n=+\infty} \frac{N_{n} G_{n}(\rho)}{n Q_{n}}\left(1-n \frac{\Omega}{\omega}\right) \cdot e^{i n \varphi} .
\end{gathered}
$$

In order to obtain an expression for the current density in the relative form we select the basic value of the current density. Let us assume that the base value is equal to

$$
\delta_{b}=\frac{W I}{\pi R^{2}} .
$$

Then

$$
\hat{\delta}(\rho, \varphi)=\frac{\delta(\rho, \varphi)}{\delta_{b}}
$$

Electromagnetic torque impacting the winding of the inductor, is defined by the following expression:

$$
\begin{aligned}
M_{e m} & =R \cdot \operatorname{Re}\left\{l \sum_{m=1}^{M} \int_{\varphi_{m}-\Delta \varphi_{m}}^{\varphi_{m}+\Delta \varphi_{m}} \mu_{0} \dot{H}_{\rho 2}(R, \varphi) J_{m}^{*} R \partial \varphi\right\}= \\
& =2 \frac{R^{2} l \mu_{0}}{\pi} j^{2} \operatorname{Re}\left\{\sum_{n=-\infty}^{n=+\infty} \frac{N_{n 1} N_{n 2}}{n^{2}} \frac{G_{n}(R)}{Q_{n}}\right\} .
\end{aligned}
$$

Expressions for the electromagnetic power can be recorded in relative units. As the base moment we adopt the moment defined by the expression

$$
M_{b}=\frac{P_{b}}{2 \tau f} R=\mu_{0} l 2 \tau R j^{2}
$$

Then the relative rotating electromagnetic moment is equal to

$$
\hat{M}_{b}=\frac{\hat{M}_{e m}}{M_{b}}=\frac{R}{\pi \tau} \operatorname{Re}\left\{\sum_{n=-\infty}^{n=+\infty} \frac{N_{n 1} N_{n 2}}{n} \frac{G_{n}(R)}{Q_{n}}\right\} .
$$

\section{DISCUSSION RESULTS}

In the beginning, we assume that magnetic core of radius $\mathrm{R}$ fully covers the cylindrical channel of radius $\mathrm{r}$ with the liquid metal. The magnetic core bears six $(M=6)$ coils of
3 -phase windings with phase areas of $60^{\circ}$ electric. In this case we have an ordinary induction motor with a massive aluminium rotor and closed magnetic core. For the purposes of calculation, the following values were adopted $\hat{R}=1.1 ; \varepsilon=2 ;$ sliding $\mathrm{s}=0.3$. Fig. 3 presents relations of a relative value of the current density $\hat{\delta}$ from angle $\varphi$ at different values of the relative radius $\hat{\rho}$. On the surface of the metal $(\hat{\rho}=1)$ the current density repeats the pattern of current density distribution in the inductor, with a bigger current density opposite the slots with currents and lower current density opposite teeth. With the removal from the surface $(\hat{\rho}<1)$ this effect decreases.

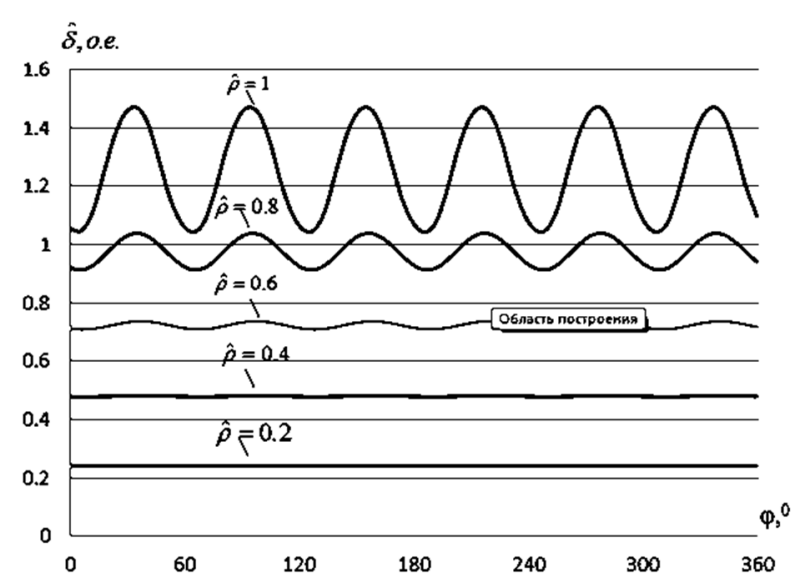

Fig. 3. The relation of the current density to the angle at different value of the relative radius.

Fig.4 presents the relation of the relative moment $\hat{M}$ to sliding $\mathrm{s}$ with different values of $\mathrm{Q}$ factor $\mathrm{q}=\varepsilon 2,3,6$. At $\varepsilon=2$ we have classic characteristic of induction motor with maximum starting torque $(\mathrm{s}=1)$ [11]. When $\varepsilon$ increases (for example, with the increase in the frequency of the supply voltage) the mechanical characteristic changes, the maximum torque is achieved at lower values of sliding. [12]

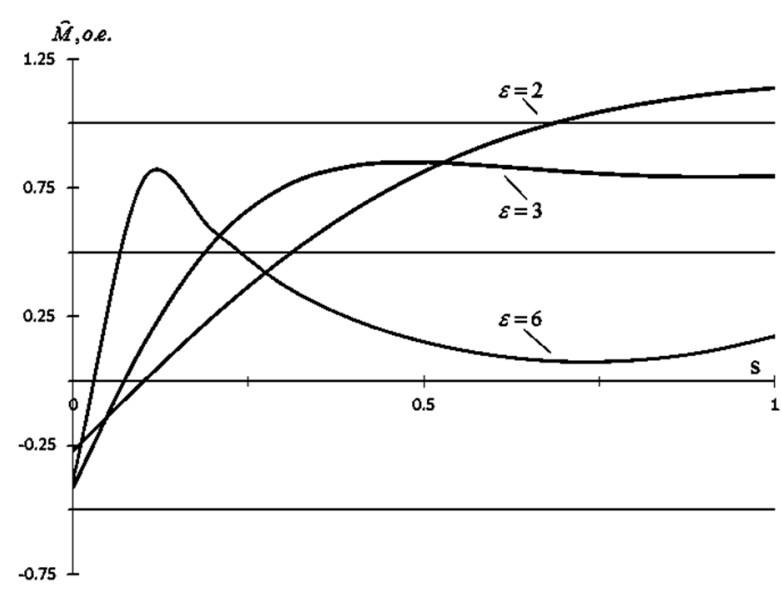

Fig. 4. Relation of the relative moment to sliding at different $\mathrm{Q}$ factor of $\varepsilon$. 
Fig.5 and 6 show the relation of the current density $\hat{\delta}$ to the angle $\varphi$ and relative moment $\hat{M}$, to sliding $\mathrm{s}$ when six $(\mathrm{M}=6)$ three-phase winding coils are located on the half of length of a magnetic core $2 \pi R$.

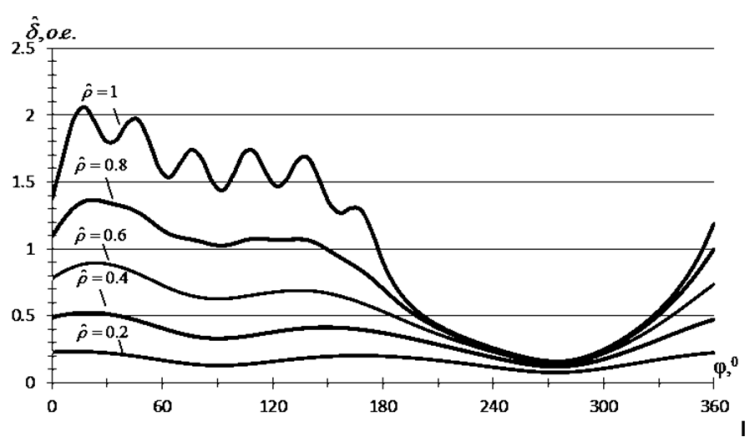

Fig. 5. The relation of the current density to the angle at different value of the relative radius for MHD rotator.

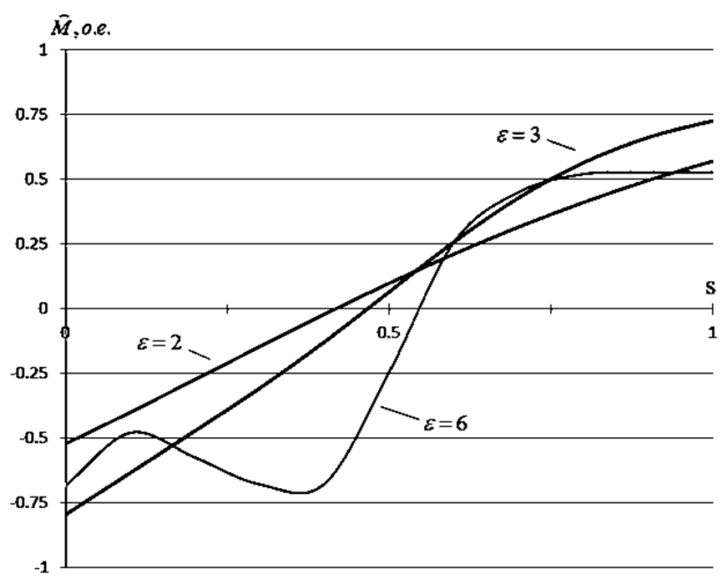

Fig. 6. Relation of the relative moment to sliding at different $\mathrm{Q}$ factor.

Here we have an open magnetic core and the unit of MHD rotator of liquid metal, which is an arc asynchronous machine with a longitudinal boundary effect. The distribution of current density becomes uneven, in addition to the slot ripples there emerge the waves of longitudinal boundary effect. These waves significantly influence the mechanical characteristic of the machine.

\section{FINDINGS AND CONCLUSIONS}

1. By adopting certain assumptions there has been built the analytical design model for the MHD rotator of liquid metal.

2. The solution for electromagnetic field vector has been obtained in the Fourier series of a complex form using Bessel functions.

3. The analytical expressions for differential and integral characteristics of MHD rotator have been obtained, the current density distribution in liquid metals has been calculated in addition to the electromagnetic power and torque at different modes of the unit operation.

4. The results obtained are used when designing a control system for kneading Rotator MHD small particles (sawdust, shavings, etc.) in the melt melting furnace with a view to enhancing the effectiveness of fusion.

\section{ACKNOWLEDGEMENTS}

This research has been completed with the financial support of the Russian Fund of Fundamental Research and the Government of Krasnoyarsk Territory within the framework of Research Project No. 18-48-242013 "Research into the impact of spatial and time-andfrequency distributions of electromagnetic forces in the melt on the nature of the MHD processes in liquid metal".

\section{REFERENCES}

[1] G.F. Shemetev, Aluminium alloys: compositions, properties, application, Student book on the course "Production of ingots from alloys of nonferrous metals", Saint-Petersburg State Polytechnical University, Saint-Petersburg, 2012.

[2] Klaus Malpol, Rudolf Hillen. Using returns profitably. Processing of aluminum chip scrap at the enterprise. http://www.ruscastings.ru/work/168/2130/2132/5517

[3] Fdhila RB, Sand U, Eriksson JE, et al. A stirring history. ABB Review. 2016;3:45-48.

[4] S. Denisov, V. Dolgikh, S. Khripchenko, I. Kolesnichenko, I. Nikulin, The effect of travelling and rotating magnetic fields on the structure of aluminum alloy during its crystallization in a cylindrical crucible // Magnetohydrodynamics Vol. 50, No. 4, 407-422, 2014

[5] Umbrashko, A., Baake, E., Nacke, B.and Jakovics, A. Modeling of the turbulent flow in induction furnaces, Metallurgical and Materials Transactions B 37B, . - 2016.pp. 831- 838

[6] V. N. Timofeev Transverse edge effect in MHD-stirrers for liquid metals / V. N. Timofeev, M. Y. Khatsayuk, M.A. Eremin // Advanced Engineering Forum. - 2016. - T. 17. - C. 66-76

[7] Kirpo M., Jakovics A., Baake E., Nacke B. LES study of particle transport in turbulent recirculated liquid metal flows // Magnetohydrodynamics.. No. 3. pp. 439-450, 2009.

[8] S. Yu. Khripchenko, R. R. Siraev, S. A. Denisov, V. M. Dolgikh , I V. Kolesnichenko, Liquid metal flow exposed to modulated travelling and rotating magnetic fields in a cylindrical crucible, Vol. 54, No. 4, 373-382, 2018

[9] Timofeev V.N. Electromagnetic rotators, stirrers and dosers of aluminum melts, the thesis of Doctor of Technical Sciences: 05.05.03 Krasnoyarsk, 1994.- 413 p.

[10]Andre Ango. Mathematics for electrical and electronic engineers. Moscow: 1965

[11]Timofeev V.N., Khatsayuk M.Y. Analysis of electromagnetic processes magnetohydrodynamic stirring of liquid metals. Electricity, 2017, No. 1, pp. 35-44.

[12] Voldek A.I. Inductive magnetic hydrodynamic machine with liquidmetal working body. - Leningrad: Energiya, 1970. - 272 p.

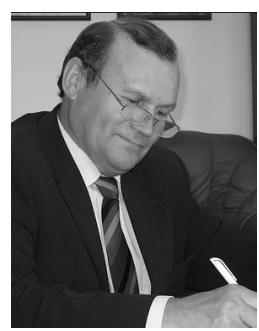

Viktor N. Timofeev was born in 1950, in the Ermolayevo Village, Balakhta District, Krasnoyarsk Territory. In 1974, he completed his education in Krasnoyarsk Polytechnical Institute majoring in Power Plants. In 1978, he was awarded the degree of the Candidate of Technical Sciences. In 1994, he was awarded the degree of the Doctor of Technical Sciences.

Area of expertise: electromagnetic fields analysis; research into electrical process equipment and MHD technology for metallurgical purposes; linear electric machines; methods of complex mathematical and physical simulation; computer aided design of electrical process equipment.

He is the member of the Thesis Board for awarding titles of Candidate and Doctoral degree, the author of over 200 scientific publications, 
including 2 monographs, 8 educational books, and 70 inventor's certificates and patents. Since 1990, he has been heading the "Research and Production Center of Magnetic Hydrodynamics", Ltd ("RPC Magnetic Hydrodynamics" Ltd), which designs and supplies electrical process equipment and technology to smelters.

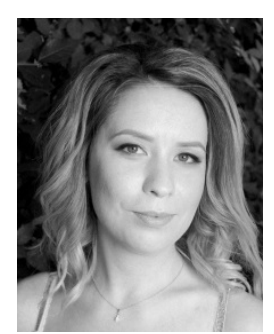

Natalia S. Shakhoval was born in the Solenoozernoye Village, Krasnoyarsk Territory. In 2015, she received Master's Degree, Siberian federal University. She has presently completed the third year of post-graduation studies. Starting from 2016, she is the Teaching Assistant at the department of Electric Technologies in Metallurgy, Siberian federal University. She is the author of 15 articles. Area of expertise: MHD technology in metallurgy, innovations in the field of magnetic hydrodynamics, electromagnetic fields analysis

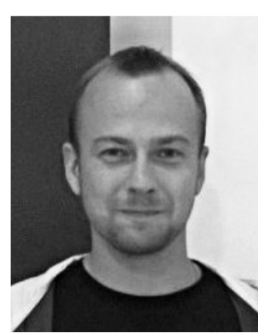

Maksim Y. Khatsayuk was born in 1988 in Minusinsk Town, Krasnoyarsk Territory. He was qualified as an Engineer in specialty of "Electric process units and systems" in the Siberian Federal University (Krasnoyarsk) in 2010, and was awarded the degree of Candidate of Technical Sciences specializing in "Electric machines" in the Ural Federal University (Yekaterinburg) in 2014.

In 2014 - 2018, he held the position of Teaching Assistant, Senior Lecturer, Associate Professor of the Electrical Equipment and Electrical Process Technology Department in the Polytechnic Institute of the Siberian Federal University. In $2010-2018$, he is an Engineer, Researching Engineer, Head of the Mathematical Simulation Department at "RPC Magnetic Hydrodynamics" Ltd. He is the author of 4 methodological and educational books, 65 publications, 12 patents in the field of electrical process technology and MHD-equipment for metallurgy, acted as a Manager and Executor of 14 R\&D projects. Area of expertise: research into related electromagnetic, thermal, magnetic and hydrodynamic processes in metallurgical units with a view to obtain materials of new physical-mechanical properties and enhanced safety, environment and energy efficiency of the electrical process equipment.

Prize winner of the contest held by the Krasnoyarsk City Mayor, the State Award of the Krasnoyarsk Territory, Award of the Strategic Research Fund "Siberian Club", Award of the IFC Bank in scientific nominations, medal of All-Russian Exhibition of Scientific and Technical Creativity of Youth "For achievements in the scientific and technical creativity".

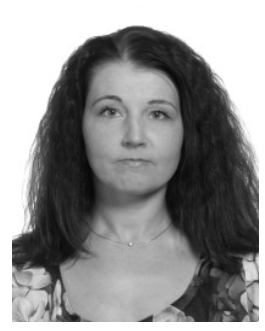

Svetlana V. Kurnaeva was born in Zvenigovo Mari-El in 1978. She received Master's Degree the Siberian Federal University in 2015 (Krasnoyarsk, Russia).

In 2015, she received Master's Degree at the department of Electric Technologies in Metallurgy, Siberian federal University. Svetlana has presently completed the third year of post-graduation studies.

She is the author of 12 publications. Area of expertise: magnetic and hydrodynamic processes and equipment for metallurgical designation.

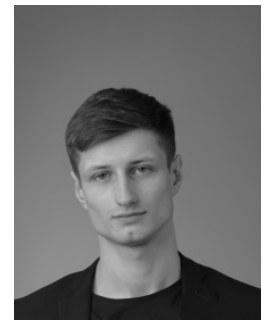

Eduard R. Vinter was born in Severomuysk, Republic of Buryatia, in 1995. In 2016, he received a Master's Degree in Electrical Engineering in Metallurgy at the Siberian Federal University (Krasnoyarsk). Since 2016, he is laboratory assistant at the Power Electronics Department at "RPC Magnetic Hydrodynamics" Ltd. Since 2018, he is assistant of the Electrical Equipment and Eletrical Process Technology Department in the Polytechnic Institute of the Siberian Federal University. Area of expertise: study of electromagnetic, thermal and hydrodynamic processes in magnetohydrodynamic installations of metallurgical purpose. Participant of international scientific and technical exhibitions and conferences. He is the author of 6 publications. 